\title{
Hábitos de uso de Internet en niños y niñas de 8 a 12 años: un estudio descriptivo
}

\author{
Alicia Peñalva Vélez ${ }^{1}$ \\ https://orcid.org/0000-0001-7396-3922 \\ María Napal Fraile ${ }^{2}$ \\ https://orcid.org/0000-0002-1058-9395 \\ Universidad Pública de Navarra, España
}

Internet use habits in children age 8 to 12: a descriptive study

Recibido: 15-05-2019

Aceptado: 31-07-2019

\section{Cita Recomendada}

Peñalva, A. \& Napal, M. (2019). Hábitos de uso de Internet en niños y niñas de 8 a 12 años: un estudio descriptivo. Hamut'ay, 6(2), 55-68.

http://dx.doi.org/10.21503/hamu.v6i2.1775

\section{RESUMEN}

La irrupción de la Web 2.0 ha supuesto la aparición de nuevas formas de producir, distribuir, intercambiar y recibir información a través de medios electrónicos. En este contexto, es necesario alfabetizar a la población para que sea capaz de hacer un uso eficaz de las redes de comunicación como vía habitual de interrelación, y esto incluye enseñar a cómo usar de manera segura Internet, a través de una adecuada configuración de la identidad personal en el mundo digital. Este trabajo tiene como objetivo investigar las características del uso de Internet de escolares de Educación Primaria, en 4 dimensiones (Uso de dispositivos y acceso a Internet, Actividad en la red, Control parental y Seguridad e identidad digital), mediante un cuestionario de alfabetización digital integrado por 74 ítems y en el que incluye una quinta dimensión, la de ciberconvivencia, que no es analizada en este estudio. Los resultados basados en las respuestas de 290 niños y nińas de $3^{\circ}$ a $6^{\circ}$ de Primaria, permiten constatar que el acceso a Internet está muy extendido, desde las primeras edades, principalmente a través de un móvil propio. El uso de Internet y los dispositivos electrónicos se relacionan sobre todo con contextos y actividades de ocio, y herramientas sociales. En términos generales, el alumnado considera su identidad y reputación, aunque no tanto su privacidad, e incurre en más conductas inseguras en cursos más altos. El control parental es escaso en todos los niveles, lo que sugiere la pertinencia de proponer programas específicos de alfabetización digital, que deberían atender a los rasgos específicos del uso de Internet en cada sexo y grupo de edad.

Palabras Clave: uso seguro de Internet, identidad digital, alfabetización digital, ciberconducta.

\footnotetext{
${ }^{1}$ Profesora contratada del Departamento de Ciencias Humanas y de la Educación (área de Didáctica de las ciencias experimentales) de la universidad Pública de Navarra. Email: Alicia.penalva@unavarra.es

${ }_{2}^{2}$ Profesora contratada del Departamento de Ciencias Humanas y de la Educación (área de Didáctica y Organización Escolar) de la universidad Pública de Navarra. Email: maria.iapal@unavarra.es
} 


\section{Abstract}

The irruption of Web 2.0 has led to the appearance of new ways to produce, distribute, exchange and receive information through electronic means. In this context, it is necessary to provide digital literacy to the population so that it is able to make effective use of communication networks as a usual way of interrelation; and this includes teaching how to safely use the Internet, through an adequate configuration of the personal identity in the digital world. This work aims to examine the characteristics of Internet use of Primary School students, in 4 dimensions (Use of devices and Internet access, Network Activity, Parental Control and Security and Digital Identity). We used a digital literacy questionnaire composed of 74 items, which includes a fifth dimension related to cyber-coexistence, which is not analyzed in this study. The results based on the responses of 290 boys and girls from 3rd to 6th grade of primary school, allow us to verify that the Internet access is widespread, from very early ages, mainly through their own mobiles. The use of the Internet and electronic devices are mostly related with leisure contexts and activities, and social media tools. In general terms, students consider their identity and reputation, although not so much their privacy, and are more likely to engage in unsafe behaviors in higher courses. Parental control is weak at all levels, suggesting the relevance of proposing specific digital literacy programs, which should address the specific features of Internet use in each sex and age group.

Keywords: safe Internet use, digital identity, digital literacy, cyber-conduct.

\section{INTRODUCCIÓN}

Las Tecnologías de la Información y la comunicación (TIC) son la vía más utilizada para el procesamiento y transmisión de gran parte de la información que se maneja en todos los ámbitos y edades (Castells, 2005). La comunicación en tiempo real y simultánea entre varios sujetos a través de un ordenador, las comunidades humanas virtuales o las redes sociales, los mensajes y correos escritos a través de la telefonía móvil o de Internet... todo ello está modificando, como señalan Ortega, Casas \& Del Rey (2014) la forma en la que se relacionan niños y niñas de distintas edades. La irrupción de la Web 2.0 ha supuesto la aparición de nuevas prácticas sociales, comunicativas y relacionales, que implican nuevas formas de producir, distribuir, intercambiar y recibir información a través de medios electrónicos (Lankshear \& Knobel, 2009).

Estas prácticas dan forma a los procesos comunicativos y socializadores característicos del ámbito digital. Emplean códigos expresivos y acciones comunicativas específicas, y diferenciadas de la comunicación a través de la escritura y la lectura en documentos de papel (Bautista, 2007). El hecho de que ocupen un espacio tan importante en los procesos de socialización y comunicación de las personas (Malo \& Figuer, 2010; Pettit, 2009;), hace necesaria una formación o alfabetización específica (Area, Borrás \& San Nicolás, 2015). Como afirman González-Fernández \& Salcines-Talledo (2015):

Las condiciones casi ilimitadas de acceso a todo tipo de información mediante el uso de las tecnologías emergentes (...) deberían llevar parejos sistemas de entrenamiento para su selección, contraste y valoración. Es decir, una educación paralela que trabaje la percepción, recepción, comprensión, análisis y reflexión sobre informaciones de toda índole. Al igual que una formación transversal que favorezca la comunicación y el trabajo en equipo (...). De este modo se favorecerían generaciones de ciudadanos cultos, críticos y creativos adaptados a la Sociedad del Conocimiento (p.2). 
Nos encontramos ante lo que Castells (2005) denomina sociedad de la información, enmarcada, como afirma Lévy (2007) en su respectiva cibercultura. Nuestra cultura es multimodal (Area, 2015), y necesita que los procesos de alfabetización de la ciudadanía se planifiquen más allá de la mera formación en herramientas tecnológicas. Hemos pasado de una cultura "solida" propia del siglo XIX a una cultura "líquida” característica del siglo XX, en la que el conocimiento es completamente inestable y fluye a cada momento (Bauman, 2006). La cultura digital, líquida y multimodal, plantea a la ciudadanía la necesidad de: (1) aprender a manejar los aparatos y su software, (2) desarrollar competencias o habilidades cognitivas relacionadas con la obtención, compresión y elaboración de la información, y (3) cultivar y desarrollar actitudes y valores que den sentido y significado ideológico, moral y político a las actuaciones desarrolladas con la tecnología (Area, 2015).

En este sentido se hace necesario recurrir a Area (2015) cuando se refiere al concepto de multialfabetizaciones o alfabetizaciones múltiples. Este autor centra su atención en cómo se pueden adquirir y dominar destrezas centradas en el uso personal, social y cultural de múltiples herramientas y lenguajes tecnológicos de representación. Pero no únicamente como habilidades instrumentales de utilización de las distintas tecnologías, sino, básicamente, como instrumentos de interacción social. En el contexto de sociedad informacional la ciudadanía se convierte en ciudadanía digital o ciberciudadanía (León, 2013; Pineda, 2011), definida como aquella que es capaz de: hacer un uso eficaz de las redes de comunicación como vía habitual de comunicación, y usar Internet como vía de participación democrática.

Pineda (2011, p. 165) indica que:

Siguiendo a las profesoras Mossber, Tolber \& McNeal, ciudadanía digital "is the ability to participate in society online", es decir, es la capacidad de participar en una sociedad conectada (online), así que el ciudadano digital es aquel que utiliza Internet regular y eficazmente de forma cotidiana. Esto se concreta en tres aspectos: el uso eficaz de las redes de comunicación para comunicarse de forma regular, la capacidad de usar Internet para participar como ciudadanos democráticos y la influencia de Internet en la igualdad de oportunidades en el ámbito económico.

Internet es una herramienta fundamental para la población de todas las edades (Alvarez-García, García, Cueli \& Núñez, 2019) y ofrece posibilidades múltiples tanto para el aprendizaje como para las relaciones sociales (Marques, T., Marques, A. \& Alvarez, 2016). Pero también supone riesgos relacionados con su uso inadecuado (Fernández-Montalvo, Peñalva \& Irazabal, 2015). El papel de los adultos en este sentido resulta fundamental (Peñalva-Vélez, Leiva \& Irazabal, 2017), tanto el del profesorado (Peñalva-Vélez, Napal \& Mendioroz, 2018) como el de la familia (Álvarez-García, Barreiro-Collazo, Núñez \& Dobarro, 2016). Sin embargo, como señalan Livingstone \& Helsper (2008) o Alvarez-García et al. (2019), según se avanza en edad existe una tendencia a controlar menos el uso que los niños y jóvenes hacen de Internet. Por ello, resulta cada vez más necesario incidir en los procesos de alfabetización digital (Muñoz, Sádaba \& Naval, 2011).

\section{Alfabetización digital}

La alfabetización para la cultura digital debe centrarse no tanto en las habilidades de uso de la tecnología, como en el proceso de adquisición y dominio de las destrezas centradas en el uso de la información y la comunicación (consumidas y/o producidas) (Rangel \& Peñalosa, 2013; Avello \& López, 2015; Fernández-Montalvo et al., 2015). La alfabetización digital favorece que cada persona sepa construirse una identidad digital como ciudadano autónomo, culto y democrático en la red, (Area et al., 2015). La alfabetización digital debe tener en cuenta además todos los procesos relacionales que, vinculados con la convivencia, se ponen en marcha en el mundo digital. En este sentido Ortega et al. (2014) inciden claramente en el hecho de que "la vida social de los escolares (...) tiene en el dispositivo digital para la comunicación una ampliación del escenario directo en el cual, hasta ahora, acontecía el proceso relacional que se ha denominado convivencia" (p.616).

Por lo tanto, cualquier programa de alfabetiza- 
ción digital debe tener en cuenta la definición de Area et al. (2015), cuando la define como "construcción de una identidad digital como persona culta, autónoma, crítica y con valores democráticos en los entornos culturales de Internet" (p.26). $Y$ en el sentido que la definen Gionés-Valls \& Serrat-Brustenga (2010), como aquella que consiste en enseñar a cómo usar de manera segura Internet a través de una adecuada configuración de la identidad personal en el mundo digital. Es decir, los programas de alfabetización digital deben partir de tres conceptos teóricos: el concepto de web 2.0, el concepto de alfabetización digital y el concepto de Identidad Digital definido por Gionés-Valls \& Serrat-Brustenga (2010).

La Web 2.0 ha cambiado las reglas de juego establecidas en el ámbito de las relaciones comunicativas basadas en los medios de comunicación convencionales (Area \& Pessoa, 2012). A partir del desarrollo de la Web 2.0 la comunicación deja de basarse en un emisor que se dirige a muchos receptores aislados entre sí. Cada persona puede actuar como un medio de comunicación, creando sus propios blogs, participando en redes sociales, difundiendo y utilizando recursos.... (Area \& Pessoa, 2012). Las nuevas aplicaciones y técnicas que ofrece, son el punto de partida para un nuevo concepto de aprendizaje y de comunicación, un nuevo concepto de socialización. Implica unas habilidades técnicas mínimas, pero unas importantes habilidades de manejo de los contenidos, puesto que el usuario es el que crea sus propios contenidos (Cebrián, 2008).

Como indican Area \& Pessoa (2012) la Web 2.0 implica distintos escenarios o ámbitos de aprendizaje: biblioteca, mercado de servicios, puzzle de contenidos, espacio público de comunicación, escenario de expresión multimedia, entorno de experiencias virtuales. De cada uno se pueden extraer otras seis dimensiones en las que los sujetos deben ser alfabetizados para un uso seguro de la Web 2.0 (Figura 1):

\begin{tabular}{|c|c|}
\hline \multicolumn{2}{|r|}{$\begin{array}{l}\text { Las dimensiones o ámbitos alfabetizadores ante las nuevas formas culturales } \\
\text { de la Web } 2.0\end{array}$} \\
\hline $\begin{array}{l}\text { Aprender a usar la Web } \\
2.0 \text { como una biblioteca } \\
\text { universal }\end{array}$ & $\begin{array}{l}\text { Esla dimensión de la alfabetización surge como respuesta a la complejidad del acceso a las } \\
\text { nuevas fuentes bibliográficas distribuidas en bases de datos digitales. Se pretende desarrollar } \\
\text { las competencias y habilidades para saber buscar información en función de un propósito dado, } \\
\text { localizarla, seleccionarla, analizarla, y reconstruirla. Es la alfabetización informacional }\end{array}$ \\
\hline $\begin{array}{l}\text { Aprender a usar la Web } \\
2.0 \text { como un mercado de } \\
\text { servicios }\end{array}$ & $\begin{array}{l}\text { Esta dimensión de alfabetización tiene que ver con la formación critica del consumidor y del } \\
\text { ciudadano. La compra de productos o la realización de gestiones administrativas online requiere } \\
\text { no solo tener las habilidades de adquisición y pago, sino también formarse como trabajador y } \\
\text { consumidor consciente de sus derechos y responsabilidades en la Red }\end{array}$ \\
\hline $\begin{array}{l}\text { Aprender a usar la Web } \\
2.0 \text { como un puzle de } \\
\text { microcontenidos } \\
\text { interenlazados }\end{array}$ & $\begin{array}{l}\text { Esta dimensión en su papel de alfabetización se dirige a la capacitación del sujeto como } \\
\text { individuo que sabe navegar de forma consciente por la Red de un documento o unidad } \\
\text { informativa a otra, que es capaz de reinterpretar y construir su propia narrativa de significados a } \\
\text { partir de unidades básicas de contenidos que, aparentemente, están separados, pero que el } \\
\text { sujeto les otorga un discurso. En definitiva, que domina las formas hipertextuales de } \\
\text { organización de la información tanto como consumidor como productor de mensajes culturales }\end{array}$ \\
\hline $\begin{array}{l}\text { Aprender a usar la Web } \\
2.0 \text { como espacio público } \\
\text { de comunicación en redes } \\
\text { sociales }\end{array}$ & $\begin{array}{l}\text { Esta dimensión de la alfabetización se refiere a la capacidad de participar de forma plena en } \\
\text { comunidades o grupos humanos interconectados a través de redes de telecomunicaciones y, en } \\
\text { consecuencia, desarrollar comportamientos sociales basados en la colaboración e intercambio } \\
\text { de información compartida }\end{array}$ \\
\hline $\begin{array}{l}\text { Aprender a usar la Web } \\
2.0 \text { como un espacio de } \\
\text { expresión multimedia y } \\
\text { audiovisual }\end{array}$ & $\begin{array}{l}\text { Esta dimensión de la alfabetización se desarrolla con la finalidad de formar al alumnado como } \\
\text { sujeto con capacidad para analizar y producir textos en formato multimedia y lenguaje } \\
\text { audiovisual Persigue formar a los sujetos tanto para el consumo critico de los productos } \\
\text { audiovisuales, así como para su producción, publicación y difusión a través de los entornos } \\
\text { digitales. Es la alfabetización multimedia v/o audiovisual }\end{array}$ \\
\hline $\begin{array}{l}\text { Aprender a usar la Web } \\
2.0 \text { como un territorio de } \\
\text { experiencias virtuales } \\
\text { interactivas }\end{array}$ & $\begin{array}{l}\text { Esta dimensión de la alfabetización supone disponer de las habilidades para interaccionar en } \\
\text { entornos virtuales tridimensionales de realidad inmersiva, simulada o aumentada. Requiere } \\
\text { adquirir habilidades de interacción no solo con la máquina, sino también con la información y } \\
\text { con otros humanos en tiempo real y/o diferido para tomar decisiones inteligentes }\end{array}$ \\
\hline
\end{tabular}

Figura 1. Dimensiones o ámbitos alfabetizadores de la Web 2.0 (Area \& Pessoa, 2012). 
La irrupción de la Web 2.0 ha supuesto la aparición de nuevas prácticas sociales que implican nuevas formas de producir, distribuir, intercambiar y recibir textos, todo ello a través de medios electrónicos (Lankshear \& Knobel, 2009). La alfabetización digital consiste en enseñar a cómo usar de manera segura Internet, a través de una adecuada configuración de la identidad personal en el mundo digital (Gionés-Valls \& Serrat-Brustenga, 2010). Ambos conceptos de alfabetización inciden en un aspecto básico de las TIC, el relativo a su uso como herramientas de comunicación y gestión de la información (Gutiérrez, Palacios \& Torrego, 2010). Es un concepto que entra dentro de la categoría conocida como "nuevas alfabetizaciones". Esta expresión se refiere al uso eficaz de Internet no tanto para saber desenvolverse a nivel instrumental en el medio, como por saber gestionar de un modo seguro todos los datos que se transmiten y reciben a través de este (De Pablos, 2010).

\section{Identidad Digital Personal}

La Identidad Digital personal se define como la habilidad para gestionar con éxito la propia visibilidad, reputación y privacidad en la red (Gionés-Valls \& Serrat-Brustenga, 2010; Area et al., 2015). La visibilidad es toda aquella actividad que genera un individuo en la red, positiva o negativa, autoconstruida o fruto de referencias o comentarios de terceros. La reputación recae en la opinión que otras personas tienen de un sujeto, aunque también puede construirla en parte el propio sujeto. La privacidad se entiende como una pieza clave para la gestión de la identidad digital, puesto que es necesario que el sujeto sea consciente de la necesidad de mantener la privacidad de los datos personales en Internet, así como que sea consciente del uso que se puede hacer de estos datos.

No se puede obviar que la educación formal y obligatoria afronta en estos momentos, entre otros, un reto fundamental: la alfabetización de los nińos y niñas para la cultura digital y la ciudadanía digital (Berzosa, 2015; Sánchez-Antolín \& Paredes, 2014; Del Rey, Casas \& Ortega, 2012; Gutiérrez, 2007). Niños y niñas desde las primeras edades viven lo que Malo \& Ferrer (2010) identifican como "infancia de los medios". Medios audiovisuales y electrónicos desempeñan un papel muy relevante en su actividad diaria, determinando y definiendo sus experiencias, y la forma en que se interiorizan. Efectivamente, tal y como sostienen Gil, Vall-Llovera \& Feliu (2010), el consumo de Tecnologías de la Información y la Comunicación (TIC) forma parte de nuestra cotidianeidad y se encuentra intrínsecamente asociada a las personas de todas las edades, y a su entorno relacional. En este sentido resulta necesario tener en cuenta a autores como Pettit (2009); Area (2015), cuando afirman que las TIC son una instancia socializadora de la infancia, al igual que lo son la familia, los pares y la escuela.

Esto, unido al hecho de que tanto los nativos digitales como los inmigrantes digitales (Premsky, 2010) usan las TIC, y más concretamente la Red (Internet), de manera habitual. Pero es la población infantil, adolescente y juvenil la que más rápida y ampliamente se ha digitalizado en sus hábitos frente a la población adulta.

Por todo lo expuesto hasta el momento, el objetivo de este estudio es describir qué hábitos de uso de Internet tiene una muestra de estudiantes de 8 a 12 años, su percepción del control que ejercen los adultos sobre su actividad en Internet, y establecer líneas prioritarias de atención a la hora de diseñar una intervención educativa de alfabetización digital para niños y niñas desde las primeras edades.

\section{Materiales Y Métodos}

\section{Participantes}

En el estudio participaron 290 alumnos y alumnas de tres centros de Educación Infantil y Primaria de la provincia de Navarra (Espańa), entre los cursos de $3^{\circ}$ a $6^{\circ}$ de Educación Primaria (Tabla 1 ).

\section{Instrumento}

Para la realización de este estudio se ha empleado un cuestionario ad hoc, de elaboración propia, al que se ha denominado "cuestionario de alfabetización digital”. El cuestionario tiene el formato de un autoinforme, y los ítems que lo componen 
Tabla 1

Distribución por sexo y curso de la muestra

\begin{tabular}{lrrrrl}
\hline & 30 & 40 & 50 & 60 & TOTAL \\
\hline Niños & 42 & 41 & 36 & 29 & 148 \\
Niñas & 36 & 35 & 32 & 36 & 139 \\
NA & 1 & & 1 & 1 & \\
\hline Total & 79 & 76 & 69 & 66 & 290 \\
\hline
\end{tabular}

Fuente: Elaboración propia (2019)

se agrupan en un total de cinco dimensiones: uso de dispositivos electrónicos e Internet, actividad en Internet y control parental en el uso de Internet, seguridad y configuración de la identidad digital, ciberconvivencia. El cuestionario incluye únicamente cuestiones cerradas: verdadero o Falso, opción múltiple o escala Likert de 4 niveles (nada, poco, bastante, mucho).

El cuestionario fue validado (contenido y constructo) mediante validación de jueces expertos (Robles Garrote \& del Carmen Rojas, 2015). Cuatro expertos no implicados en la investigación, con dilatada trayectoria investigadora en el campo de la alfabetización mediática, confirmaron la exhaustividad y pertinencia de las cuestiones, así como la precisión, legibilidad y adecuación a los destinatarios de las preguntas.

El valor de alfa de Cronbach global para el cuestionario completo fue 0.91 , lo que indica una alta consistencia interna. Igualmente, las cuatro dimensiones consideradas mostraron también alta consistencia interna. Los datos analizados en el estudio corresponden con las cuatro primeras dimensiones del cuestionario señaladas (Tabla 2).

Tabla 2

Dimensiones analizadas a través de los instrumentos de evaluación, cuestiones referidas a la dimensión y $\alpha$ de Cronbach

\begin{tabular}{lcc}
\hline Dimensiones analizadas & Items & $\begin{array}{c}\text { a Cron- } \\
\text { bach }\end{array}$ \\
\hline $\begin{array}{l}\text { Dimensión 1. Uso de dispositivos elec- } \\
\text { trónicos e Internet }\end{array}$ & $1-13$ & 0.76 \\
$\begin{array}{l}\text { Dimensión 2. Actividad en Internet } \\
\text { Dimensión 3. Control parental en el uso } \\
\text { de Internet }\end{array}$ & $41-53$ & 0.81 \\
$\begin{array}{l}\text { Dimensión 4. Alfabetización e identidad } \\
\text { digital }\end{array}$ & $54-64$ & 0.74 \\
\hline
\end{tabular}

Fuente: Elaboración propia (2019)
Todos los análisis estadísticos se han desarrollado en RStudio (Versión 1.0.136 - (C) 2009-2016 RStudio, Inc.), basado en $\mathrm{R}$ versión 3.6.0 ( $\mathrm{R}$ Core Team, 2019), y los paquetes coin (Hothorn, 2008) y psych (Revelle, 2018)

\section{Resultados}

A continuación, se exponen los resultados de la investigación, los cuales se distribuyen en función de las cuatro dimensiones definidas en la Tabla 2. Se comienza el análisis con los resultados referidos a la Dimensión 1 (Uso de dispositivos y acceso a Internet).

Tal y como nos indican los datos (Tabla 3) el alumnado que dispone de teléfono móvil va aumentando de manera progresiva con la edad, y alcanza un $70 \%$ en el curso $5^{\circ}$ y un $85 \%$ en el curso $6^{\circ}$. Se observa una evolución relevante en cuanto a uso de dispositivos electrónicos en función de la edad. Si durante las primeras edades el porcentaje de uso de la Tablet es muy alto, disminuye conforme aumenta la edad, para dejar paso de manera prioritaria al móvil.

La universalidad de uso de los dispositivos queda reflejada en el hecho de que, sobre todo en las primeras edades, en caso de no disponer de dispositivo electrónico propio, se usa en un alto porcentaje el de otra persona de la familia.

Respecto al teléfono móvil, destaca el hecho de que la mayoría de alumnos y alumnas que disponen de este aparato también tienen acceso a Internet, en todas las edades o cursos. No existe diferencia por sexos en ninguna de las cuestiones.

\section{Tabla 3}

Porcentaje del alumnado (\%) en posesión de dispositivos electrónicos (Móvil, Tablet, Ordenador) con acceso a Internet, por cursos $\left(3^{\circ}\right.$ a $\left.6^{\circ}\right)$. Se acompaña del valor del test $\mathrm{X}^{2}$ para la diferencia entre cursos $\left(\mathrm{X}^{2} \mathrm{y} p\right)$

\begin{tabular}{|c|c|c|c|c|c|c|}
\hline & $\begin{array}{c}30 \\
(n=79)\end{array}$ & $\begin{array}{c}4^{\circ} \\
(n=76)\end{array}$ & $\begin{array}{c}5^{\circ} \\
(n=69)\end{array}$ & $\begin{array}{c}60 \\
(n=66)\end{array}$ & $X^{2}$ & $p$ \\
\hline \multicolumn{7}{|c|}{ Tengo mi propio: } \\
\hline $\begin{array}{l}\text { Teléfono } \\
\text { móvil }\end{array}$ & 34.6 & 40.8 & 69.6 & 84.8 & 49.04 & 0.00 \\
\hline Tablet & 69.6 & 61.8 & 56.5 & 53.0 & 6.72 & 0.08 \\
\hline Ordenador & 30.4 & 23.7 & 30.4 & 37.9 & 3.16 & 0.37 \\
\hline
\end{tabular}




\begin{tabular}{lcccccc}
\hline & $\begin{array}{c}3^{\circ} \\
(\mathrm{n}=79)\end{array}$ & $\begin{array}{c}4 \circ \\
(\mathrm{n}=76)\end{array}$ & $\begin{array}{c}5^{\circ} \\
(\mathrm{n}=69)\end{array}$ & $\begin{array}{c}6^{\circ} \\
(\mathrm{n}=66)\end{array}$ & $X^{2}$ & $p$ \\
\hline $\begin{array}{l}\text { Como no dispongo de uno propio, utilizo el de otra perso- } \\
\text { na de la familia: }\end{array}$ \\
$\begin{array}{l}\text { Teléfono } \\
\text { móvil }\end{array}$ & 54.4 & 35.5 & 33.3 & 21.2 & 15.15 & 0.00 \\
Tablet & 21.5 & 13.2 & 24.6 & 18.2 & 4.91 & 0.18 \\
Ordenador & 49.4 & 44.7 & 49.3 & 54.5 & 2.98 & 0.39 \\
\hline $\begin{array}{l}\text { Dispone de conexión a Internet: } \\
\text { Teléfono }\end{array}$ & 36.7 & 36.8 & 68.1 & 78.8 & 42.04 & 0.00 \\
móvil & & & & & & \\
Tablet & 63.3 & 63.2 & 50.7 & 54.5 & 2.39 & 0.50 \\
Ordenador & 39.2 & 47.4 & 55.1 & 68.2 & 14.11 & 0.00 \\
\hline
\end{tabular}

Fuente: Elaboración propia (2019)

En la Tabla 4 se observa que el uso del teléfono móvil supera al de la Tablet y el ordenador. No se puede desligar el uso de Internet del uso del móvil, y ambos aumentan de manera significativa según avanzan los cursos. Según informan los sujetos de la muestra, los fines de semana o días de fiesta se utilizan los dispositivos más que los días de colegio, incluido el ordenador. No existe diferencia por sexos en ninguna de los usos.

Tabla 4

Intensidad del acceso a Internet y uso de dispositivos electrónicos, por cursos. La intensidad de uso se calcula como promedio de las puntuaciones en Likert

\begin{tabular}{lcccccc}
\hline & $\begin{array}{c}30 \\
(n=79)\end{array}$ & $\begin{array}{c}\text { 4o } \\
(n=76)\end{array}$ & $\begin{array}{c}5^{\circ} \\
(n=69)\end{array}$ & $\begin{array}{c}60 \\
(n=66)\end{array}$ & $X^{2}$ & $p$ \\
\hline Uso Internet & 1.6 & 1.7 & 1.7 & 2.1 & 24.02 & 0.00 \\
\hline Los días de colegio: & & & & & \\
Uso Internet & 1.1 & 0.8 & 0.9 & 1.2 & 20.13 & 0.02 \\
Uso el móvil & 0.6 & 0.6 & 0.8 & 1.2 & 39.94 & 0.00 \\
Uso Tablet & 0.8 & 0.5 & 0.5 & 0.5 & 16.07 & 0.07 \\
Uso el & 0.7 & 0.5 & 0.4 & 0.9 & 21.78 & 0.01 \\
ordenador & & & & & & \\
\hline Los fines de semana o días de fiesta: & & & \\
Uso Internet & 1.6 & 1.5 & 1.9 & 2.2 & 35.23 & 0.00 \\
Uso el móvil & 1.4 & 1.3 & 1.9 & 2.1 & 34.18 & 0.00 \\
Uso Tablet & 1.4 & 1.2 & 1.3 & 0.9 & 15.87 & 0.07 \\
Uso el & 1.2 & 1.0 & 0.9 & 1.1 & 8.58 & 0.48 \\
ordenador & & & & & & \\
\hline
\end{tabular}

Fuente: Elaboración propia (2019)

En la Tabla 5 se puede observar que el uso de Internet también se ha ido adelantando: así, en el espacio sólo de cuatro años (cuatro cursos aca- démicos), la edad de inicio en el uso de Internet ha retrocedido más de un año. Así, existe una correlación positiva y significativa entre la edad del alumnado y la edad de inicio ( $\mathrm{rho}=0,469$; $\mathrm{p}<$ 0.001). Si comparamos entre sexos, la brecha $(0,8$ años entre los alumnos y alumnas de $6^{\circ}$ ) desaparece entre los más jóvenes (Tabla 5).

Tabla 5

Edad (Media (SD)) de inicio en el uso de Internet

\begin{tabular}{ccccc}
\hline & $3^{\mathrm{o}}$ & $4^{\mathrm{o}}$ & $5^{\mathrm{o}}$ & $6^{\mathrm{o}}$ \\
\hline Masculino & $6.9(1.09)$ & $7.8(1.29)$ & $7.5(1.25)$ & $8.3(1.85)$ \\
Femenino & $6.9(1.01)$ & $7.9(1.4)$ & $8.6(1.5)$ & $9.1(1.85)$ \\
\hline
\end{tabular}

Fuente: Elaboración propia (2019)

En la Tabla 6 se exponen los resultados relativos a cómo perciben los niños y niñas su competencia para el uso de Internet en comparación con la de los adultos (padre, madres, profesorado). En su mente la brecha digital es una realidad, visto que casi la mitad de los escolares de $5^{\circ}$ y $6^{\circ}$ considera que sabe bastante o mucho más que sus padres o madres. El porcentaje disminuye cuando se refiere a los profesores y profesoras, aunque en ambos casos aumenta con el curso. No hay diferencias significativas por sexo.

\section{Tabla 6}

Porcentaje de alumnado con un conocimiento de Internet mejor $^{1}$ que el de sus adultos de referencia. Se incluye el valor del test $\mathrm{X}^{2}$ para la diferencia entre cursos $\left(\mathrm{X}^{2}\right.$ y $\left.\mathrm{p}\right)$

\begin{tabular}{lcccccc}
\hline & $\begin{array}{c}30 \\
(n=79)\end{array}$ & $\begin{array}{c}4^{\circ} \\
(n=76)\end{array}$ & $\begin{array}{c}5^{\circ} \\
(n=69)\end{array}$ & $\begin{array}{c}6^{\circ} \\
(n=66)\end{array}$ & $X^{2}$ & $p$ \\
\hline $\begin{array}{l}\text { Sé más cosas sobre Internet: } \\
\begin{array}{l}\text { Que mi padre } \\
\text { o madre }\end{array}\end{array}$ & 38.0 & 21.1 & 43.5 & 42.4 & 23.02 & 0.01 \\
$\begin{array}{l}\text { Que mis } \\
\text { profesores o } \\
\text { profesoras }\end{array}$ & 7.6 & 9.2 & 7.2 & 16.7 & 30.71 & 0.00 \\
\hline
\end{tabular}

${ }^{1}$ Porcentaje (\%) de alumnado que ha seleccionado las categorías 2 (bastante) o 3 (mucho).

Fuente: Elaboración Propia (2019)

En las Figuras 2 y 3 se analizan los datos referidos a las aplicaciones de Internet que usan los niños y niñas de manera habitual, según informan ellos mismos. Como se ve en la Figura 2, entre las aplicaciones utilizadas por el alumnado de $2^{\circ}$ y 3er ciclo de Primaria destacan especialmente la aplicación de mensajería WhatsApp, YouTube e 
Instagram. Muy por debajo, con menos de $10 \%$ de usuarios frecuentes, tenemos otras aplicaciones sociales. Esto incluye el uso del e-mail, que se supone medio de comunicación privilegiado en situaciones virtuales de aprendizaje.

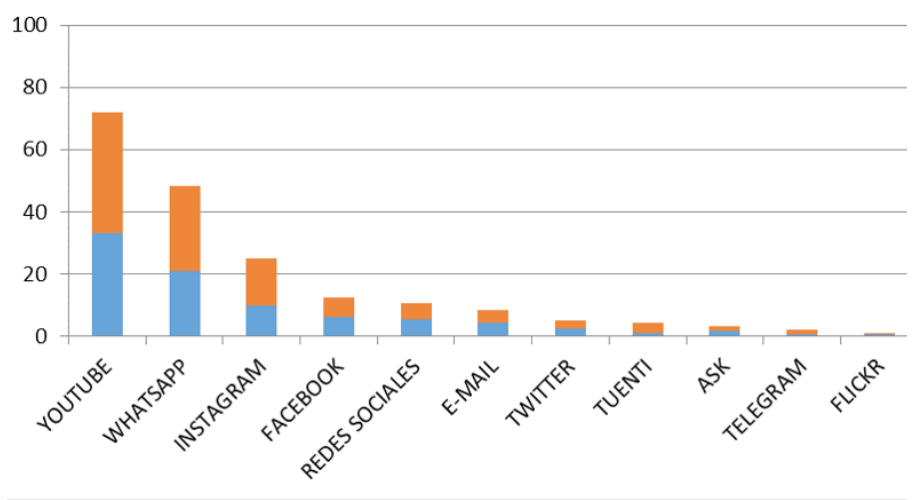

Figura 2

Porcentaje de usuarios que utilizan bastante (azul) o mucho (naranja) las diversas aplicaciones.

Fuente: Elaboración Propia (2019).

En la Figura 3 se comprueba que en el caso de las 3 aplicaciones más frecuentes (YouTube, WhatsApp e Instagram, en este orden), se produce un aumento paulatino, pero especialmente destacable en $6^{\circ}$, y en el caso de los varones. De hecho, ambos sexos difirieron en su uso de YouTube $(\mathrm{X} 2=10,93 ; \mathrm{df}=3 ; \mathrm{p}=0.01226)$, WhatsApp $(\mathrm{X} 2=7,2081 ; \mathrm{df}=3 ; \mathrm{p}=0.06555)$ e Instagram $(\mathrm{X} 2=6,0732 ; \mathrm{df}=3 ; \mathrm{p}=0.1081)$.

La Dimensión 2, relativa a la actividad que desarrollan los sujetos de la muestra en Internet, analiza datos que definen a los usuarios como productores y/o consumidores de contenidos. $\mathrm{La}$ actividad más frecuente, según informan los niños y niñas, es el consumo de productos de ocio (vídeos de youtubers y música, especialmente), seguida por la búsqueda de información y el juego e interacción social. En estas interacciones sociales, según la percepción de los alumnos y alumnas, con poca frecuencia se producen conductas de riesgo o inadecuadas (Tabla 7).

En general, no se observa una notable evolución por cursos, ni diferencias significativas por sexos en estas actividades. La excepción más notable son los videojuegos, individuales o en red con otras personas, y el visionado de vídeos sobre videojuegos, más comunes entre los niños (Tabla 8).
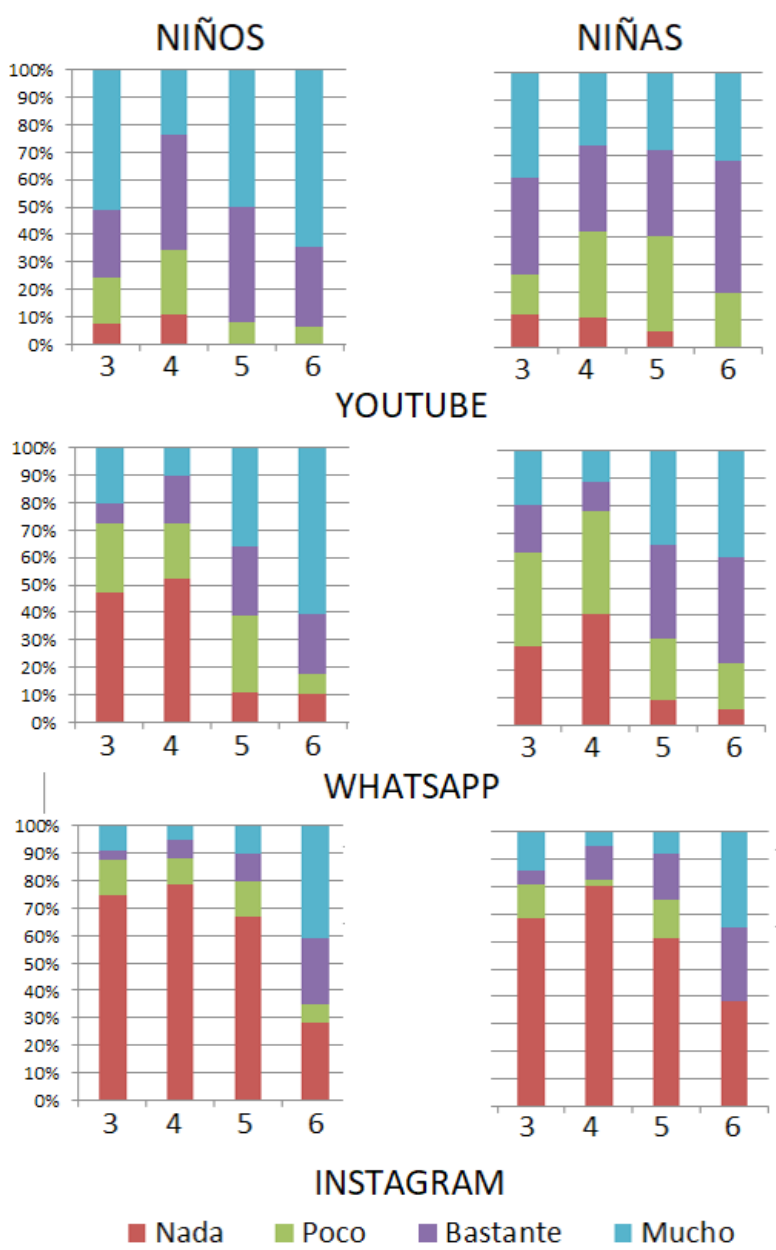

Figura 3

Evolución con los cursos (3o-6o) en el uso de las aplicaciones más frecuentes, por sexos.

Tabla 7

Actividades en Internet. Porcentajes por categoría (0: nada; 1: poco; 2: bastante; 3: mucho). Se sombrean las categorías más frecuentes

NA $\quad \begin{array}{llll}0 & 1 & 2 & 3\end{array}$

\section{Consumo ocio:}

1. Escucho música

$\begin{array}{lllll}1.0 & 9.7 & 31.4 & 27.9 & 30.0\end{array}$

2. Veo videos sobre jugue- $\quad \begin{array}{lllll}1.0 & 31.7 & 35.2 & 20.7 & 11.4\end{array}$ tes o canciones

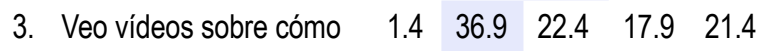
jugar a juegos, o vídeos sobre cómo juegan otros

4. Veo vídeos de youtubers $1.0 \quad 19.3 \quad 17.9 \quad 22.4 \quad 39.3$

$\begin{array}{lllllll}\text { 11. Descargo música, } & 2.1 & 34.1 & 27.9 & 17.9 & 17.9\end{array}$ películas, fotos...

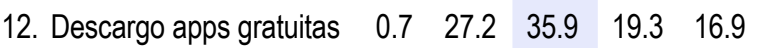
o de pago (aplicaciones o juegos de play store)

\section{Información:}

$\begin{array}{lllllll}\text { 7. Busco información } & 2.8 & 12.4 & 37.2 & 30.3 & 17.2\end{array}$

10. Uso información para las $\begin{array}{llllll}2.4 & 25.2 & 41.0 & 19.0 & 12.4\end{array}$ tareas del colegio 


\begin{tabular}{|c|c|c|c|c|c|}
\hline & NA & 0 & 1 & 2 & 3 \\
\hline \multicolumn{6}{|l|}{ Juego - Ficción } \\
\hline $\begin{array}{l}\text { 8. Juego solo o sola o } \\
\text { contra el ordenador }\end{array}$ & 1.4 & 45.9 & 28.3 & 13.8 & 10.7 \\
\hline $\begin{array}{l}\text { 9. Juego con otras perso- } \\
\text { nas en juegos on line }\end{array}$ & 1.7 & 55.2 & 18.6 & 12.8 & 11.7 \\
\hline $\begin{array}{l}\text { 19. Paso el tiempo en un } \\
\text { mundo virtual }\end{array}$ & 0.7 & 52.4 & 33.4 & 9.0 & 4.5 \\
\hline $\begin{array}{l}\text { 20. Creo personajes, o } \\
\text { mascotas o avatares }\end{array}$ & 1.4 & 74.8 & 12.1 & 7.6 & 4.1 \\
\hline \multicolumn{6}{|l|}{ Social } \\
\hline $\begin{array}{l}\text { 5. Visito los perfiles de } \\
\text { otras personas en redes } \\
\text { sociales }\end{array}$ & 2.1 & 67.2 & 20.7 & 5.5 & 4.5 \\
\hline $\begin{array}{l}\text { 6. Mando mensajes con } \\
\text { WhatsApp u otros }\end{array}$ & 2.1 & 32.8 & 23.4 & 22.8 & 19.0 \\
\hline 14. Visito chats & 2.4 & 66.6 & 19.3 & 7.2 & 4.5 \\
\hline $\begin{array}{l}\text { 15. Visito páginas web de } \\
\text { otras personas }\end{array}$ & 0.3 & 73.8 & 16.9 & 4.5 & 4.5 \\
\hline $\begin{array}{l}\text { 16. Publico mensajes sobre } \\
\text { cosas o personas }\end{array}$ & 1.0 & 82.1 & 12.4 & 2.4 & 2.1 \\
\hline \multicolumn{6}{|l|}{ Produzco } \\
\hline $\begin{array}{l}\text { 13. Publico o cuelgo } \\
\text { música, películas, fotos, } \\
\text { vídeos }\end{array}$ & 0.3 & 67.6 & 21.7 & 4.8 & 5.5 \\
\hline $\begin{array}{l}\text { 17. Uso páginas para } \\
\text { compartir ficheros (fotos, } \\
\text { vídeos, música...) }\end{array}$ & 1.7 & 70.0 & 19.0 & 6.9 & 2.4 \\
\hline 18. Uso la webcam & 1.4 & 75.5 & 13.1 & 5.9 & 4.1 \\
\hline 21. Creo blogs & 0.7 & 89.7 & 6.6 & 2.8 & 0.3 \\
\hline $\begin{array}{l}\text { 22. Hago fotos o grabo } \\
\text { vídeos }\end{array}$ & 0.7 & 39.3 & 35.5 & 14.5 & 10.0 \\
\hline \multicolumn{6}{|l|}{ Seguridad } \\
\hline $\begin{array}{l}\text { 23. Respondo a mensajes } \\
\text { de personas que no } \\
\text { conozco }\end{array}$ & 1.4 & 85.9 & 7.6 & 2.4 & 2.8 \\
\hline $\begin{array}{l}\text { 24. Quedo con personas } \\
\text { que no conozco para } \\
\text { vernos en la vida real }\end{array}$ & 0.7 & 93.1 & 3.8 & 2.1 & 0.3 \\
\hline $\begin{array}{l}\text { 25. Cuelgo fotos de otras } \\
\text { personas sin su permiso }\end{array}$ & 1.0 & 93.1 & 5.2 & 0.0 & 0.7 \\
\hline $\begin{array}{l}\text { 26. Veo cosas molestas o } \\
\text { me hace sentir incómo- } \\
\text { do/a o disgustado/a }\end{array}$ & 0.3 & 73.8 & 17.9 & 6.2 & 1.7 \\
\hline $\begin{array}{l}\text { 27. Me molestan otras per- } \\
\text { sonas con comentarios } \\
\text { sobre mí }\end{array}$ & 0.3 & 74.5 & 12.4 & 5.9 & 6.9 \\
\hline
\end{tabular}

Fuente: Elaboración Propia (2019)
Tabla 8

Actividades en la red en las que se manifiestan diferencias por sexos. Valores como promedio de las puntuaciones en Likert

\begin{tabular}{lcccc}
\hline & $\begin{array}{c}\text { Niño } \\
(148)\end{array}$ & $\begin{array}{c}\text { Niña } \\
(139)\end{array}$ & $X^{2}$ & $p$ \\
\hline 4. Veo vídeos de youtubers & 2.01 & $(139)$ & 61.90 & 0.00 \\
3. Veo vídeos sobre cómo & 1.74 & 0.73 & 13.13 & 0.00 \\
$\begin{array}{l}\text { jugar a juegos, o vídeos } \\
\text { sobre cómo juegan otros }\end{array}$ & & & & \\
$\begin{array}{l}\text { 8. Juego solo o sola contra } \\
\text { el ordenador }\end{array}$ & 1.07 & 0.70 & 14.99 & 0.00 \\
$\begin{array}{l}\text { 9. Juego con otras personas } \\
\text { en juegos online }\end{array}$ & 1.13 & 0.44 & 36.39 & 0.00 \\
\hline
\end{tabular}

Fuente: Elaboración Propia (2019)

En la Dimensión 3, referida al control parental de los padres y madres sobre sus hijos e hijas, se observa que, cuando usan Internet la familia tiene, globalmente, poca presencia (Tabla 9). Sí prohíben ciertas actividades $\mathrm{o}$, con menor frecuencia, controlan lo que los niños hacen en Internet. Raramente acompañan al menor mientras utiliza Internet o dialogan sobre ello. En contraste, parece claro que ofrecen pautas para desarrollar conductas adecuadas y seguras en Internet. Tampoco existen en esta dimensión diferencias en función del sexo. No hay tampoco diferencia en función del curso, lo que indica que el control parental no es más intenso en los cursos inferiores.

\section{Tabla 9}

Control y acompañamiento por adultos (familia). Porcentajes por categoría (0: nada; 1 : poco; 2: bastante; 3: mucho). Las celdas sombreadas indican el valor más frecuente (en el caso de diferencias $<2.5 \%$ se señalan varias celdas)

\begin{tabular}{|c|c|c|c|c|c|c|}
\hline & & NA & 0 & 1 & 2 & 3 \\
\hline 1. & $\begin{array}{l}\text { Hablan contigo sobre lo } \\
\text { que haces en Internet }\end{array}$ & 0.7 & 32.1 & 33.4 & 17.9 & 15.9 \\
\hline 2. & $\begin{array}{l}\text { Se sientan contigo mien- } \\
\text { tras usas Internet viendo } \\
\text { lo que haces pero sin } \\
\text { meterse }\end{array}$ & 1.0 & 46.6 & 29.0 & 11.7 & 11.7 \\
\hline 3. & $\begin{array}{l}\text { Hacen cosas contigo en } \\
\text { Internet }\end{array}$ & 1.7 & 39.7 & 33.4 & 15.2 & 10.0 \\
\hline 4. & $\begin{array}{l}\text { Te ayudan cuando } \\
\text { tienes dificultades para } \\
\text { hacer o encontrar algo } \\
\text { en Internet }\end{array}$ & 1.4 & 20.7 & 27.9 & 28.6 & 21.4 \\
\hline & $\begin{array}{l}\text { Te explican por qué } \\
\text { algunas páginas son } \\
\text { buenas o malas }\end{array}$ & 0.3 & 13.1 & 16.6 & 33.8 & 36.2 \\
\hline
\end{tabular}




\begin{tabular}{|c|c|c|c|c|c|}
\hline & NA & 0 & 1 & 2 & 3 \\
\hline $\begin{array}{l}\text { 6. Te explican cómo } \\
\text { mejorar tu seguridad en } \\
\text { Internet }\end{array}$ & 1.4 & 18.6 & 13.8 & 29.3 & 36.9 \\
\hline $\begin{array}{l}\text { 7. Te explican cómo } \\
\text { comportarte con otras } \\
\text { personas en Internet }\end{array}$ & 1.4 & 33.4 & 13.4 & 19.7 & 32.1 \\
\hline $\begin{array}{l}\text { 8. Te ayudan cuando algo o } \\
\text { alguien te ha molestado } \\
\text { en Internet }\end{array}$ & 5.2 & 35.2 & 11.0 & 20.7 & 27.9 \\
\hline $\begin{array}{l}\text { 9. Te dicen qué debes } \\
\text { hacer si algo o alguien } \\
\text { te molesta en Internet }\end{array}$ & 3.1 & 30.0 & 13.4 & 26.9 & 26.6 \\
\hline $\begin{array}{l}\text { 10. Me prohíben hacer al- } \\
\text { gunas cosas en Internet } \\
\text { (compras, descargar } \\
\text { aplicaciones, jugar a } \\
\text { juegos) }\end{array}$ & 1.7 & 18.3 & 24.8 & 25.2 & 30.0 \\
\hline $\begin{array}{l}\text { 11. Me ponen un horario de } \\
\text { uso de Internet }\end{array}$ & 0.3 & 41.0 & 18.6 & 19.0 & 21.0 \\
\hline $\begin{array}{l}\text { 12. Me controlan qué hago } \\
\text { en Internet }\end{array}$ & 0.3 & 27.9 & 22.8 & 23.4 & 25.5 \\
\hline
\end{tabular}

Fuente: Elaboración Propia (2019)

La última dimensión se refiere a la alfabetización e identidad digital; es decir, la gestión de la propia visibilidad, reputación y privacidad en la red. La Figura 4 muestra los datos personales que comparten los niños y niñas en las redes sociales: encontramos que más del $40 \%$ compartirían datos personales como fotografías privadas y el apellido, la edad real, e incluso el número de teléfono o el nombre de la escuela o colegio (aprox. 25\%).

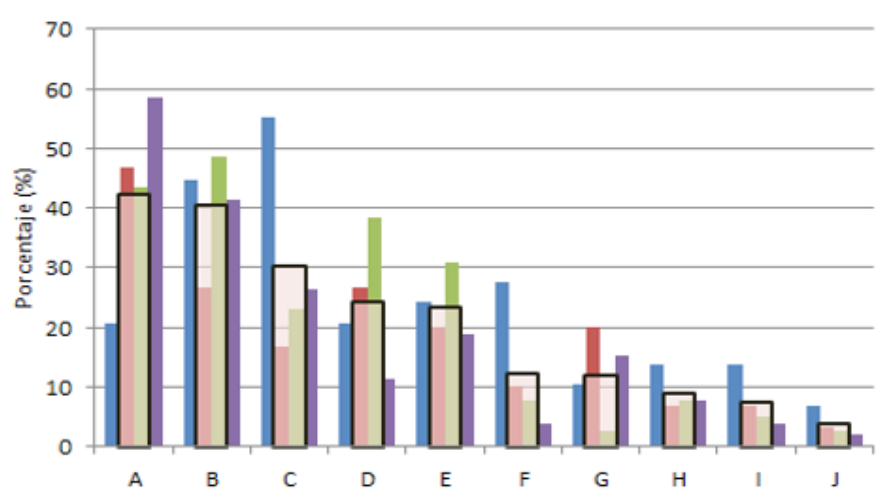

( $A=$ Foto en la que se me reconoce; $B=A$ pellidos; $C=E$ dad real; $D=E$ dad que no es la mía; $E=$ Número de teléfono; $F=$ Colegio 0 escuela; $G=$ Fotos de otros; $\mathrm{H}=$ Fotos de mi vida privada; $\mathrm{I=Dirección;} \mathrm{J=Mensajes} \mathrm{sobre} \mathrm{otras} \mathrm{peisonas).}$

Figura 4

Porcentaje del alumnado que publica cada tipo de información en sus perfiles en redes sociales. Porcentajes calculados sobre el total de respuestas válidas.

Fuente: Elaboración Propia (2019) a) Si alguien quiere ser mi amigo en una red social que yo uso (Facebook, Twitter, Instagram...

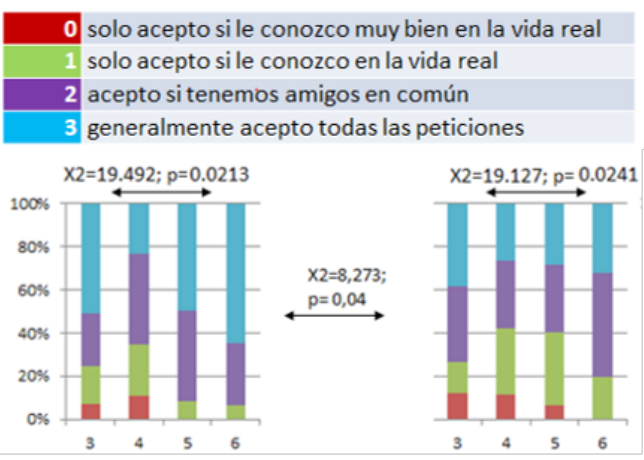

b) Cuando creo un perfil en una red social...

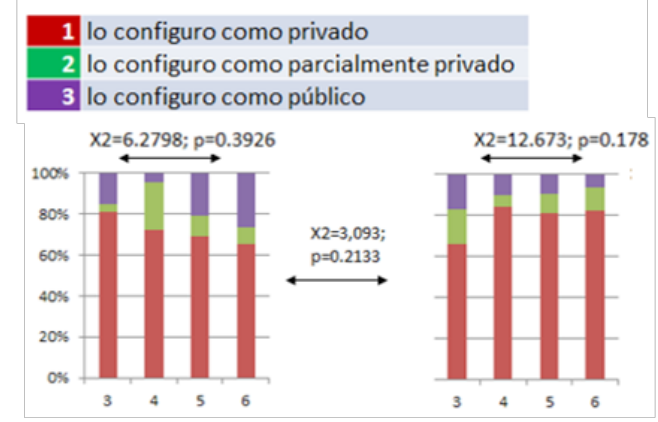

c) En mis redes sociales, si alguien me pide que le dé información personal sobre mí, o datos personales (teléfono, dirección, foto, edad real...)

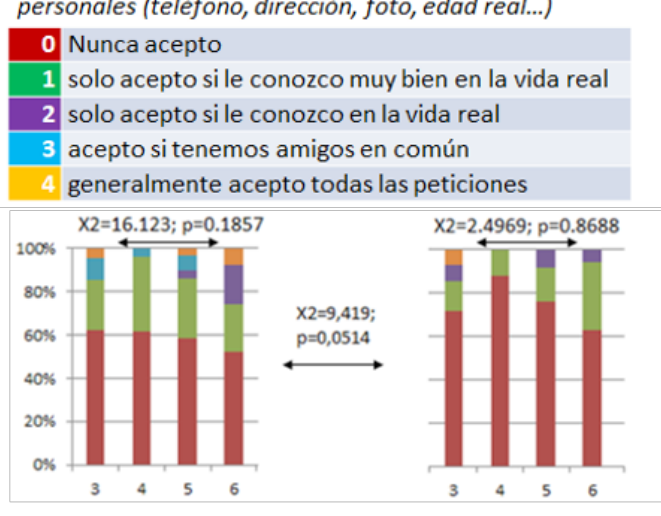

d) Cuando hablo con las personas a través de Internet o las redes sociales

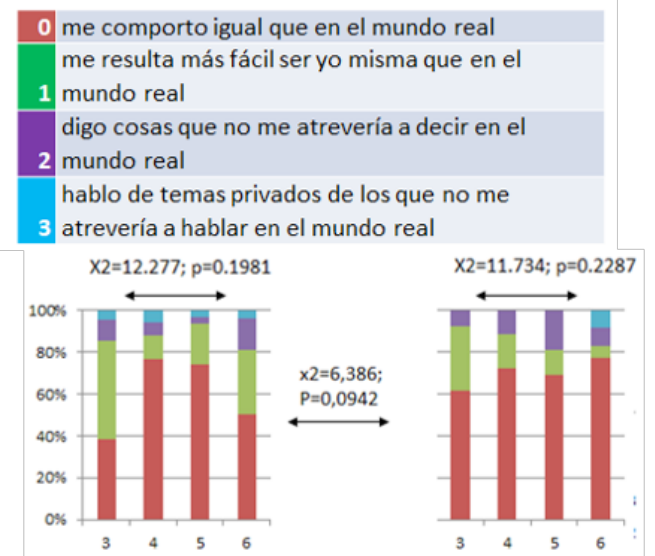

Figura 4

(a-d). Visibilidad, privacidad y reputación en la red. Porcentajes en niños (izda.) y niñas (dcha.) Para cada comparación, se proporciona el estadístico de contraste entre sexos (entre las gráficas) y entre cursos (sobre las gráficas). 
Como se evidencia en la Figura 4, los niños y niñas tienden a conductas seguras (configurar los perfiles como privados - izquierda abajo-, regirse por las mismas conductas que en el mundo real - derecha abajo-, y sólo compartir información con personas que conocen (derecha arriba). Sin embargo, hay una tendencia a conductas más inseguras en el caso de los varones, y estas se hacen más frecuentes con el paso de los cursos.

Si nos detenemos en aquellas cuestiones relacionadas con la reputación e identidad digital, vemos cómo niños y niñas lo tienen muy presente, y que esta concienciación aumenta con el paso de los cursos, siendo máxima en los alumnos y alumnas de mayor edad (Tabla 11). Ninguna de estas cuestiones muestra diferencias en función del sexo de los encuestados.

\section{Tabla 11}

Porcentaje de alumnado que considera bastante (2) o muy (3) importante la identidad y reputación digital, por curso. (Porcentajes calculados sobre las respuestas válidas)

\begin{tabular}{|c|c|c|c|c|c|c|}
\hline & $\begin{array}{c}30 \\
(n=79)\end{array}$ & $\begin{array}{c}4^{\circ} \\
(n=76)\end{array}$ & $\begin{array}{c}5^{\circ} \\
(n=69)\end{array}$ & $\begin{array}{c}60 \\
(n=66)\end{array}$ & $X^{2}$ & $p$ \\
\hline $\begin{array}{l}\text { 1. Antes de publicar } \\
\text { algo sobre mí pienso } \\
\text { en las consecuen- } \\
\text { cias (fotos, videos, } \\
\text { comentarios....) }\end{array}$ & 50.6 & 44.7 & 75.4 & 74.2 & 28.30 & 0.00 \\
\hline $\begin{array}{l}\text { 2. Antes de publicar algo } \\
\text { sobre alguien pienso } \\
\text { en las consecuen- } \\
\text { cias (fotos, vídeos, } \\
\text { comentarios....) }\end{array}$ & 48.1 & 48.7 & 87.0 & 81.8 & 32.40 & 0.00 \\
\hline $\begin{array}{l}\text { 3. En mi opinión, es } \\
\text { importante tener } \\
\text { cuidado con lo que } \\
\text { publico en Internet }\end{array}$ & 72.2 & 63.2 & 89.9 & 86.4 & 13.34 & 0.15 \\
\hline $\begin{array}{l}\text { 4. En mi opinión, es } \\
\text { importante cuidar } \\
\text { la imagen que doy } \\
\text { sobre mí en Internet }\end{array}$ & 64.6 & 57.9 & 89.9 & 75.8 & 28.30 & 0.00 \\
\hline $\begin{array}{l}\text { 5. En mi opinión, es } \\
\text { importante cuidar } \\
\text { la imagen que doy } \\
\text { sobre otras personas } \\
\text { en Internet }\end{array}$ & 59.5 & 50.0 & 89.9 & 78.8 & 32.46 & 0.00 \\
\hline $\begin{array}{l}\text { 6. En mi opinión lo que } \\
\text { publico en Internet } \\
\text { me define como } \\
\text { persona }\end{array}$ & 44.3 & 25.0 & 46.4 & 45.5 & 12.52 & 0.19 \\
\hline
\end{tabular}

Fuente: Elaboración Propia (2019).

\section{Discusión y Conclusiones}

En este estudio se ha observado que el acceso a Internet desde las primeras edades es prácticamente universal, tal y como señalan también datos de diferentes estudios (Garmendia et al. 2017, INE, 2017. La muestra de nuestro estudio así lo corrobora, y marca además una tendencia clara en relación a qué tipo de dispositivos son más comunes en función de la edad de los sujetos: en las primeras edades se prioriza el uso de la Tablet frente al ordenador, y el teléfono móvil. Pero según se avanza en la edad, es el teléfono móvil el dispositivo que se usa de manera totalmente prioritaria. La mayoría de nińos y nińas tienen un teléfono móvil que dispone de conexión a Internet (INE, 2017; Salcines - Talledo, Ramírez - García y GonzálezFernández, 2018). Resulta importante tener esto en cuenta por lo que supone la falta de control de los adultos respecto a la actividad de los niños en Internet. En estudios previos se incidía en la necesidad de ubicar los dispositivos electrónicos (en referencia al ordenador) en lugares públicos del hogar (Labrador \& Villadangos, 2009). En estos momentos estimamos que las recomendaciones deben ir más dirigidas hacia pautas para los niños y nińas sobre control de uso de los dispositivos (tiempo de uso, contenidos a los que acceden, o necesidad de desconexión) (Fernández-Montalvo et al., 2015), y que los adultos (familia y profesorado) requieren ciertas pautas para realizar un adecuado control parental (Peñalva, Leiva \& Irazabal, 2017).

La primera cuestión que emerge es una clara identificación de los dispositivos electrónicos e Internet con actividades de ocio: consumo de productos de ocio (música, vídeos de otros, búsqueda de información, no necesariamente relacionada con las tareas escolares, etc.). En esta misma línea, la actividad es sensiblemente mayor en fines de semana o días de fiesta que en los días de colegio. Este dato sugiere que el uso de Internet que hacen los niños y niñas desde las primeras edades se asocia más a un uso lúdico. Existe por lo tanto una gran área de actividad en la Red que queda fuera del control adulto, que se produce sin supervisión, y sin una formación previa (explícita y específica) de los usuarios. Atendiendo a la per- 
cepción del alumnado sobre sus conocimientos sobre Internet, comparados con los de sus padres/ madres o profesores/ profesoras, parece evidente que esta labor podría corresponder a la escuela.

En cualquier caso, un programa de estas características debe adaptarse a los perfiles de cada grupo en función de la conducta que más desarrolle en Internet, y esto puede variar en función del curso y sexo de los destinatarios. Para la mayor parte de las cuestiones analizadas no existen diferencias significativas en función del sexo: no las hay en el acceso a Internet (frecuencia y características del acceso) ni en el control parental. De hecho, parece que las diferencias existentes (en la edad de inicio en el uso de Internet) se han ido atenuando, y que las conductas de niños y niñas son cada vez más similares. La única diferencia parece estar en el uso de las aplicaciones más populares (YouTube o WhatsApp), y en las actividades relacionadas con el juego en ordenador, que son más frecuentes en nińos y en particular a partir de $5^{\circ}-6^{\circ}$ de Educación Primaria. Se aprecia también una cierta tendencia a incurrir en conductas más inseguras y con mayor frecuencia entre niños que entre niñas. En cuanto a la edad, sí se aprecia un incremento paulatino de la actividad en Internet, de modo que en 3er ciclo la inmensa mayoría de los alumnos y alumnas acceden a Internet (con frecuencia a través de dispositivos propios). Coincidiendo con esto, aumenta la actividad en aplicaciones sociales, así como su sensación de capacidad. Como ya se ha avanzado, creemos importante remarcar el uso tan bajo que se hace del ordenador, algo que estimamos interesante tener en cuenta a la hora de planificar el uso educativo de los dispositivos electrónicos, y de escoger el objetivo de las acciones formativas o preventivas.

Conviene incidir de nuevo en el hecho de que los sujetos de la muestra manifiestan que se perciben como más competentes en el uso de Internet y los dispositivos móviles que los adultos, tanto familia como profesorado. Esta idea incide en los principios básicos que parece marcar el concepto de brecha digital (Prensky, 2010), según el cual la competencia percibida por los adultos (inmigrantes digitales) es menor que la percibida por los más jóvenes (nativos digitales). Sin embargo, es necesario atender a los estudios que indican que la brecha digital no debe hacernos pensar que los denominados nativos digitales tienen competencias suficientes para el uso seguro y crítico de Internet (Rial, Gómez, Braña \& Varela, 2014). En reali$\mathrm{dad}$, teniendo en cuenta que habitualmente no existe una formación específica ni reglada de niños y nińas (ni de adultos, profesorado y familias), para el uso seguro de Internet, esta brecha digital deja de ser tal en muchos aspectos relevantes.

Sí se puede comprobar que existe un uso diferencial de redes sociales entre jóvenes y adultos tal y como ya señalan autores como (Del Rey, Mora-Merchán, Casas \& Ortega-Ruiz, 2018). La muestra analizada indica que usa de manera prioritaria tres redes: YouTube, WhatsApp e Instagram. Por edades, YouTube es la red que se usa desde edades más tempranas. Una vez que acceden al uso de dispositivos móviles se inician en el uso de WhatsApp para establecer comunicación principalmente entre los iguales, y en Instagram en las edades más superiores. En este punto sí existen diferencias entre chicos y chicas que en nuestra opinión deben ser tomadas en cuenta a la hora de plantear programas educativos de tipo preventivo y formativo.

Se puede observar que YouTube se emplea de manera muy similar en ambos sexos, y que su uso está prácticamente generalizado. El uso de WhatsApp es mínimo en las primeras edades, pero, llegados a los cursos superiores de $5^{\circ}$ y $6^{\circ} \mathrm{de}$ primaria, su uso se generaliza entre niños y niñas. El uso de Instagram se reserva también de manera principal a estos últimos cursos, y parece ser un poco más popular entre las niñas. Es muy importante remarcar la necesidad de prestar atención a la ciberconducta de los niños y niñas desde las primeras edades (Ortega et al., 2014). Algo que enlaza directamente con la ciberconviencia en el sentido que la define Ortega (2012). Los comportamientos de interacción social que ponen en marcha nińos y niñas a través de la Red deben ser un objetivo prioritario de intervención educativa, de carácter tanto formativo como preventivo (Peñalva \& Irazabal, 2017).

Por último, una dimensión que nos resulta de gran interés es la que centra la atención en el rol que desempeñan los adultos frente a las conduc- 
tas de los niños y niñas en Internet (Peñalva et al., 2017). Los sujetos de la muestra señalan que sí se sienten acompañados y orientados por los adultos, aunque de una manera insuficiente desde nuestra perspectiva. Aunque los adultos sí que marcan ciertas pautas para el uso de Internet, no lo hacen de una manera generalizada, ni acompañan a sus hijos e hijas, supervisando directamente sus actividades en la red. Esto parece deberse, según señalan diferentes estudios, a esa falta de competencia percibida que manifiestan los adultos (inmigrantes digitales) frente a los niños y niñas (nativos digitales) (Peñalva et al., 2017). En este sentido nos parece fundamental plantear planes específicos de formación para los adultos relevantes, encargados de orientar y guiar a los menores en la actividad que realizan en la Red. Esta formación debería además plantearse de manera diferencial en función de si el rol que se ejerce es el correspondiente al profesorado o a la familia (Napal, Peñalva-Vélez \& Mendioroz, 2018).

\section{REFERENCIAS BIBLIOGRÁFICAS}

Álvarez-García, D., García, T., Barreiro-Collazo, A., Dobarro, A., \& Antúnez, A. (2016). Parenting style dimensions as predictors of adolescent antisocial behavior. Frontiers in Psychology, 7, 13-83. https://doi.org/10.3389/ fpsyg.2016.01383

Álvarez-García, D., García, T., Cueli, M. \& Núñez, J.C. (2019). Control parental del uso de Internet durante la adolescencia: evolución y diferencias de género. Revista Iberoamericana de Diagnóstico y Evaluación, 51, 19-31. https://doi.org/10.21865/RIDEP51.2.02

Area, M. (2015). La alfabetización digital en la formación de la ciudadanía del siglo XXI. Revista Integra Educativa, 7 (3), 21-33.

Area, M., Borrás, J.F. \& San Nicolás, B. (2015). Educar a la generación de los Millenials como ciudadanos cultos del ciberespacio. Apuntes para la alfabetización digital. Revista de Estudios de Juventud, 109, 13-32.

Area, M. \& Pessoa, T. (2012). De lo sólido a lo líquido: Las nuevas alfabetizaciones ante los cambios culturales de la Web 2.0. Comunicar, 19, 13-20. https://doi.org/10.3916/ C38-2011-02-01

Avello, R. \& López, R. (2015). Alfabetización digital de los docentes de las escuelas de hotelería y turismo cubanas. Experiencias en su implementación. RUSC. Universities and Knowledge Society Journal, 12 (3), 1-13.

Berzosa, I. (2015). Las TIC en la escuela. Una propuesta de integración desde la investigación-acción (Tesis Doctoral). Universidad de Valladolid, Valladolid, España.

Castells, M. (2005). La era de la información: economía, sociedad y cultura: la sociedad Red. México: Alianza Editorial.

Cebrián de la Serna, M. (2008). Los procesos de innovación didáctica en el marco del Espacio Europeo de Educación Superior (EEES). El nuevo perfil del profesor universitario en el EEES: claves para la renovación metodológica. Valladolid: Universidad Europea Miguel de Cervantes, 19-36.

De Pablos, J. (2010). Universidad y sociedad del conocimiento. Las competencias informacionales y digitales. Revista de Universidad y Sociedad del Conocimiento, 7 (2), 6-16.

Del Rey, R., Casas, J.A. \& Ortega, R. (2012). El programa ConRed, una práctica basada en la evidencia. Comunicar, 39, 129-138.

Del Rey-Alamillo, R., Mora-Merchán, J.A., Casas, J.A. \& Ortega-Ruiz, R. (2018). 'Asegúrate' Program: Effects on cyber-aggression and its risk factors. Comunicar, 2018, 26 (56), 39-48. https://doi.org/10.3916/C56-2018-04

Encuesta sobre equipamientos y uso de tecnologías de la información y comunicación en los hogares. 2017. Instituto Nacional de Estadística. [Acceso: 24 junio 2018]. Disponible en: http: //www.ine.es/prensa/tych_2017.pdf.

Fernández-Montalvo, J., Peñalva, A., \& Irazabal, I. (2015). Hábitos de uso y conductas de riesgo en Internet en la preadolescencia. Comunicar, 44, 113-120. https://doi. org/10.3916/C44-2015-12

Garmendia, M. Jiménez, E., Casado, M.A. \& Mascheroni, G. (2017). Net Children Go Mobile. Risks and opportunities on Internet and the use of mobile devices amongst Spanish children (2010-2015). Bilbao.

Gil-Juárez, A., Vall-Llovera, M. \& Feliu, J. (2010). Consumo de TIC y subjetividades emergentes: ¿Problemas nuevos? Intervención Psicosocial, 19, 19-26. https://doi. org/10.5093/in2010v19n1a4

Gionés-Valls, A. \& Serrat-Brustenga, M. (2010). La gestión de la identidad digital: una nueva habilidad informacional y digital. BiD: textos universitaris de biblioteconomia i documentació, 24, 1-15.

González-Fernández, N. \& Salcines-Talledo, I. (2015). El Smartphone en los procesos de enseñanza-aprendizaje-evaluación en Educación Superior. Percepciones de docentes y estudiantes. Relieve, 21 (2), 1-20. https://doi.org/10.7203/ relieve.21.2.7480

Gutiérrez, A. (2007). Integración curricular de las TIC y educación para los medios en la sociedad del conocimiento. Revista Iberoamericana de Educación, 45, 141-156.

Gutiérrez, A., Palacios, A., \& Torrego, L. (2010). Tribus digitales en las aulas universitarias. Comunicar, 17(34).

Hothorn T, Hornik K, van de Wiel MA. \& Zeileis A (2008). "Implementing a class of permutation tests: The coin package.” Journal of Statistical Software_28 (8), 1-23. https://doi. org/10.18637/jss.v028.i08 
Labrador, F. J., \& Villadangos, S. M. (2009). Adicciones a nuevas tecnologías en jóvenes y adolescentes. En E. Echeburúa, F. J. Labrador \& E. Becoña (eds.), Adicción a las nuevas tecnologías en adolescentes y jóvenes (pp. 45-75). Madrid: Pirámide.

Lankshear, C. \& Knobel, M. (2008). Nuevos alfabetismos: su práctica cotidiana y el aprendizaje en el aula. Madrid: Ediciones Morata.

León, A. (2013). Los retos de la globalización. Migraciones y nuevas ciudadanías. Versiones, 2 (3), 47-64.

Lévy, P. (2007). Cibercultura. La cultura de la sociedad digital. México: Anthropos.

Livingstone, S., \& Helsper, E. J. (2008). Parental mediation of children's Internet use. Journal of Broadcasting \& Electronic Media, 52(4), 581-599. https://doi. org/10.1080/08838150802437396.

Malo, S. \& Ferrer, C. (2010). Infancia, adolescencia y Tecnologías de la Información y la Comunicación (TICs) en perspectiva psicosocial. Intervención Psicosocial, 19 (1), 5-8. https://doi.org/10.5093/in2010v19n1a2

Marques, T. P., Marques, A. \& Alvarez, M. J. (2016). Estudo psicométrico da Escala de Avaliação dos Riscos e Oportunidades dos Jovens Utilizadores do Facebook. Revista Iberoamericana de Diagnóstico y Evaluación - e Avaliação Psicológica, 41(1), 145-158.

Muñoz Saldaña, M.; Sádaba, Ch. \& Naval, C. (2011). La competencia mediática en los adolescentes, el caso de Internet. En Estrategias de Alfabetización Mediática: Reflexiones sobre comunicación y educación (pp. 1-16). Barcelona: Universidad Autónoma de Barcelona; Mentor Media Literacy

Napal, M.; Peñalva-Vélez, A. \& Mendióroz, A.M. (2018). Development of Digital Competence in Secondary Education Teachers' Training. Educ. Sci. 8, 104. https://doi. org/10.3390/educsci8030104

Ortega, R. (2012). Ciberconducta y relaciones en la Red: Ciberconvivencia. España: Ministerio de Educación-Universidad de Córdoba.

Ortega-Ruiz, R., Casas, J.A. \& Del Rey, R. (2014). Hacia el constructo de ciberconvivencia. Infancia y Aprendizaje, 37 (3), 602-628. https://doi.org/10.1080/02103702.2014. 957537

Peñalva Vélez, A., Napal Fraile, M. \& Mendioroz Lacambra, A.M. (2018). Competencia digital y alfabetización digital de los adultos (profesorado y familias). International Journal of New Education, 1(1). https://doi.org/10.24310/ IJNE1.1.2018.4892

Peñalva-Vélez, A. \& Irazabal, A. (2017). Digital literacy and cyberconvivencia in primary education. Procedia - Social and Behavioral Sciences, 237, 110-117. https://doi.org/10.1016/j.sbspro.2017.02.050

Peñalva-Vélez, A., Leiva, J.J. \& Irazabal, I. (2017). The role of adults in children digital literacy. Procedia-Social and Behavioral Sciences, 237, 887-892. https://doi.org/10.1016/j. sbspro.2017.02.124
Pettit, C.M. (coord.) (2009). Medios y tecnología de la información y la comunicación. Socialización y nuevas apropiaciones (TiCS). Córdoba (Argentina): Editorial Brujas.

Pineda, M. (2011). Nuevas formas de ciudadanía asociadas a las redes de comunicación globales: el ciudadano digital. Historia Actual Online, 24, 163-183.

Premsky, M. (2010). Nativos e inmigrantes digitales. Institución educativa SEK.

R Core Team (2019). R: A language and environment for statistical computing. R Foundation for Statistical Computing, Vienna, Austria. Recuperado de https://www.R-project.org/.

Rangel, A. \& Peñalosa, E.A. (2013). Alfabetización digital en docentes de educación superior: construcción y prueba empírica de un instrumento de evaluación. Píxel-Bit. Revista de Medios y Educación, 43, 9-23. https://doi.org/10.12795/ pixelbit.2013.i43.01

Revelle, W. (2018) psych: Procedures for Personality and Psychological Research, Northwestern University, Evanston, Illinois, USA. Recuperado de https:/CRAN.R-project.org/ package $=$ psych Version $=1.8 .12$.

Rial, A., Gómez, P., Brańa, T. \& Varela, J. (2014). Actitudes, percepciones y uso de Internet y las redes sociales entre los adolescentes de la Comunidad Gallega (España). Anales de Psicología, 30, 642-655. https://doi.org/10.6018/analesps.30.2.159111

Robles Garrote, P. \& Rojas, M. del. (2015) La validación por juicio de expertos: dos investigaciones cualitativas en Lingüística aplicada. Revista Nebrija de Lingüística Aplicada a la Enseñanza de Lenguas, 9(18), 124-139. https://doi. org/10.26378/rnlaeI918259

Salcines-Talledo I.; Ramírez-García A. \& González-Fernández N. (2018). Smartphones y tablets en familia. Construcción de un instrumento diagnóstico. Aula Abierta; 47 (3), 265 - 272. https://doi.org/10.17811/aula_abierta.47.3.2018.265-272

Sánchez-Antolín, P. \& Paredes, J. (2014). La concreción de las políticas educativas de integración de las TIC europeas y españolas en la Comunidad de Madrid. Teoría de la Educación y Cultura en la Sociedad de la Información, 15 (4), 107- 133 . 